\title{
Civilizing Energy Efficiency in Wireless Sensor Network Using Bacteria Foraging Algorithm
}

\author{
A.Rajeshwari ${ }^{1}$, V.Vimala Devi ${ }^{2}$, A.S.Lakshmi ${ }^{3}$, N.Nagajothi ${ }^{4}$ \\ 1, 2, 3, 4 (Assistant Professor, Department of Computer Science and Engineering) \\ 1,2,3,4 (P.S.R.Rengasamy College of Engineering for Women, Sivakasi, Tamil Nadu, India)
}

\begin{abstract}
WSN has the potentiality to join the physical world with the virtual world by creating a network of sensor nodes. Here, sensor nodes are usually battery-operated devices, and hence energy reduction of sensor nodes is a major design issue. To extend the network's lifetime, minimization of energy consumption should be used. In cluster-based routing, cluster heads shape a wireless stamina to the sink. Each cluster heads collects data starting the sensors belonging to its cluster and ahead it to the sink. Here, the cluster head position rotates, i.e., each node works as a cluster head for a restricted period of time. Energy saving in BFA approaches can be done by cluster formation, cluster-head election, data collection at the cluster-head nodes to reduce data redundancy and thus save energy and also it improve energy efficiency of homogeneous WSN. It also defined Bacterial Foraging Algorithm (BFA) as an algorithm for selecting best cluster head selection for WSN. The simulation results enhanced performance of BFA based on total energy dissipation and no.of alive nodes of the network when compared with LEACH
\end{abstract}

Keywords-WSN, BFA, LEACH, routing protocol, cluster head election.

\section{Introduction}

Wireless Sensor Networks (WSNs) consists of networked sensors that work together in hundreds of thousands of numbers for collaborative signal processing, monitoring, sensing and control tasks. WSNs offer extensive benefits and versatility to low-power and low-cost rapid deployment for many applications that can be automated without any human supervision. Many of the applications include disaster recovery, military surveillance, health administration, environmental \& habitat monitoring, target tracking. WSNs can selforganize and self-configure independently by inter-node communications possible through

multi-hop wireless paths. While the communications of each node is possible via the transceiver unit, each sensor node also consists of a sensing unit, processing unit, and a power basis unit. Each of these units is included together with the help of ICs with integrated signal processing and micro-sensing components. These nodes that form the WSN can be sited far from the actual occurrence, and can still be used for data aggregation and collection from a remote location far away from the point of event-occurrence.

This scheme uses bacteria foraging algorithm in wireless sensor network to improve the energy efficiency of each sensor nodes, whereas in previous schemes it used in control system. Nowadays Bacteria Foraging technique is gaining importance in the optimization problems. Because

- Philosophy says, Biology provides highly automated, robust and effective organism

- Search strategy of bacteria is salutary (like common fish) in nature

- Bacteria can sense, make a decision and act so adopts social foraging (foraging in groups).

To perform social foraging an animal needs communication capabilities and it gains advantages that can exploit essentially the sensing capabilities of the group, so that the group can gang-up on larger prey, persons can obtain safety from predators while in a group, and in a certain sense the group can forage a kind of intelligence. BFA is based on the foraging performance of Escherichia Coli (E. coli) bacteria present in the person intestine.

\section{Related Works}

M.Tripathy et al has proposed a new algorithm Bacteria Foraging Algorithm(BFA) along with the transformer taps are tuned with a view to simultaneously optimize the actual power losses and voltage stability limit. Kelvin M.Passino et al has proposed to improve the energy efficiency of the control system using Bacteria Foraging Algorithm (BFA). Thus extending the lifetime of the control system. Indrajit Banerjee et al has proposed to maximize the lifetime of sensor nodes and also to find the shotest path between cluster head and base station for effective data transmission to base station. Lejiang Guo,Weijiang et al has proposed to improves node energy efficiency,balances energy consumption of all sensor nodes,enhances the reliability of data transmission and increases the network lifetime in comparsion to LEACH. 
Mario A. Munoz et al has proposed some modifications to the original algorithm that simplifies the algorithm structure, and the addition of best member message into the search strategy, which improves the performance. Ben Niu et al has proposed Bacterial foraging optimization (BFA) is a fairly new bio- heuristic algorithm which is based on a metaphor of communal interaction of E. coli bacteria. Although the algorithm has successfully been practical to many kinds of actual word optimization problems, experimentation with complex problems information that the basic BFA algorithm possesses a poor performance.

Petre-Cosmin HuruiaHi et al Wireless Sensor Networks comprise of very small sensors that are characterized by limited processing power and energy resources. WSNs are used in various domains such as military applications, medical engineering, and industrial job automation. It is very important to have an best network in order to use its dispensation power at maximum. This paper proposes and analyzes the effectiveness of a hierarchical routing protocol designed to enlarge the life of the network by minimizing energy use and latency by choosing the best nodes to become cluster-heads. Minimization is realized with a multi-objective genetic algorithm performed on a central BS and the fallout send to the network nodes. Simulation is done in NS-2.

Cheng JianSheng et al,has proposed that Wireless sensor networks have attracted much research awareness in recent years and can be used in many different applications.In this paper, we analyze the impact of Energy competence in Wireless sensor networks, as a result is Clustering technique has been confirmed to be an effective approach for reducing energy consumption.It also can enlarge the scalability and lifetime of the network.

T. Datta et al,has proposed that improved adaptive approach involving Bacterial Foraging Algorithm (BFA) to improve the amplitude and phase of the weights of a linear collection of antennas for maximum array factor at any desired direction and nulls in exact directions. The Bacteria Foraging Algorithm is complete adaptive using attitude of adaptive delta modulation. Tengfei Zhu et al.,has proposed that Traditional energybalanced clustering algorithms in wireless sensor networks (WSNs) typically overlooked the different characters of energy use among clusters, which really subjective the efficiency of energy employing. To solve this issue, in this paper, a Distributed power proportional clustering (DPPC) algorithm is proposed, in which the clusters are strong-minded to be an most favorable distribution by making the total power use of the cluster proportional to the total energy it stored. In the method of cluster formation, a distributed way is adopted. The sensors firstly compare their positions and arrange themselves into the optimal clusters, then participate for the cluster heads according to their location and residual energy that are evaluated by the appropriateness function.

\subsection{Algorithm Description}

\section{Proposed Scheme}

In this scheme, BFA( bacteria foraging algorithm) is used to improve the energy efficiency in wireless sensor networks. Problem ${ }_{\text {size }}$, cells $s_{\text {num }}, n_{\text {ed }}, n_{r e}, n_{c}, n_{s}$, step $_{\text {size }}, d_{\text {attract }}, w_{\text {attract, }}, h_{\text {repellent }}, w_{\text {repellent }}, p_{\text {ed }}$ are given as a input. Algorithm output produced the best cell value where it can be a cluster head. At initial step cell number, problem size are given to find the initial population. Based on this population the high energy node is calculated. There are four basic steps, such as chemotaxis, swarming, reproduction, removal and dispersal.

$$
P(j, k, l)=\{\theta i(j, k, l) \mid i=1,2, \mathrm{~K}, S\}
$$

Let $\mathrm{j}$ be the chemotactic step, $\mathrm{k}$ be the reproduction step, 1 be the elimination and dispersal event, $\mathrm{s}$ be the bacteria. Chemotactic is a process of finding a nearby nodes. Let $N c$ be the distance end to end of the lifetime of the bacteria as deliberate by the number of chemotactic steps they take during their life.

Let $\mathrm{C}(\mathrm{i}) \lambda 0, i, \lambda 1,2, \mathrm{~K}, S$, denote a basic chemotactic step size is to define the lengths of steps during runs. Tumbling is a process of moving to nearby nodes based on the specific set of directions. To signify a tumble, a unit length arbitrary direction, say $\lambda(j)$, is generated; unit length will be used to define the path of movement after a tumble. In particular, we let

$$
\lambda i(j 1, k, 1) \lambda \lambda i(j, k, 1) C(i) \lambda(j)
$$

So that C(i ) is the size of the step taken in the arbitrary direction specified by the tumble. Swimming is same as tumbling but it doesn't have a specific set of directions only there is a little displacement. If at $\theta i$ ( $j+1, k, l)$ the cost $J(i, j+1, k, l)$ is better (lower) than at $\theta i(j, k, l)$, then another step of size C(i ) in this same direction will be taken, and again, if that pace resulted in a position with a better cost value than at the previous step, another step is taken. This swim is continued as long as it continues to reduce the cost, but only up to a utmost number of steps, Ns . This represents that the cell will be apt to keep moving if it is headed in the path of increasingly favorable environments. 


\subsection{Steps in Algorithm}

Chemo taxis: This process in the control system is achieved through swimming and tumbling via Flagella. Each flagellum is a left-handed coil configured so that as the support of the flagellum (i.e., where it is connected to the cell) rotates counterclockwise, as viewed from the free end of the flagellum looking in the direction of the cell, it produces a force against the bacterium so it pushes the cell. On the other hand, if they rotate clockwise, each flagellum pulls on the cell, and the net result is that each flagellum operates relatively independently of others, and so the bacterium tumbles about. Therefore, an E. coli bacterium can go in two different ways; it can run (swim for a period of time) or it can tumble, and exchange flank by these two modes of operation in the entire lifetime. To represent a tumble, a unit distance end to end arbitrary direction, $\operatorname{say}(j)$, is generated; this will be used to define the way of movement after a tumble. In particular

$$
\theta^{i}(j+1, k, l)=\theta^{i}(j, k, l)+c(i) \phi(j)
$$

Where $\mathrm{j}+1, \mathrm{k}, 1$ represents the $\mathrm{i}^{\text {th }}$ bacterium at $\mathrm{j}^{\text {th }}$ chemotactic step, $\mathrm{k}^{\text {th }}$ reproductive step and $\mathrm{l}^{\text {th }}$ elimination and dispersal step. c(i) is the size of the step taken in the random direction specified by the tumble (run length unit).

\section{Swarming}

When a collection of E. coli cells is located in the center of a semisolid agar with a single nutrient chemo-effecter (sensor), they shift out from the center in a traveling ring of cells byshifting up the nutrient gradient formed by consumption of the nutrient by the group. In addition to that, if high levels of succinate are used as the food, then the cells let loose the attractant aspartate so that they assemble into groups and, hence, move as a patterns of groups with high bacterial density. The spatial order results from outward movement of the ring and the local releases of the attractant; the cells provide an magnetism signal to each other so they swarm together. The mathematical representation for swarming can be represented by

$$
\begin{aligned}
& J_{c c}(\theta, P(j, k, l))=\sum_{i=1}^{S} J_{c c}^{i}\left(\theta, \theta^{i}(j, k, l)\right) \\
& =\sum_{i=1}^{S}\left[-d_{\text {attrac } \tan t} \exp \left(-w_{\text {attrac } \tan t} \sum_{m=1}^{p}\left(\theta_{m}-\theta_{m}^{i}\right)^{2}\right)\right] \\
& +\sum_{i=1}^{S}\left[h_{\text {repellent }} \exp \left(-w_{\text {attrac } \tan t} \sum_{m=1}^{p}\left(\theta_{m}-\theta_{m}^{i}\right)^{2}\right)\right]
\end{aligned}
$$

where $_{\mathrm{cc}}(\theta, \mathrm{P}(\mathrm{j}, \mathrm{k}, \mathrm{l}))$ is the cost function value to be added to the real cost function to be minimized to present a time changeable cost function, $S$ is the total number of bacteria, $P$ is the numeral of parameters to be optimized which are present in each bacterium, and $d_{\text {attract }}, \mathrm{w}_{\text {attract }}, \mathrm{h}_{\text {repellent }}, \mathrm{w}_{\text {repellent }}$ are different coefficients that are to be chosen properly.

\section{Reproduction}

The minimum healthy bacteria die and the other healthier bacteria each split into two bacteria, which are located in the same location. This makes the inhabitants of bacteria constant.

\section{Elimination and Dispersal}

It is probable that in the local environment, the lives of a population of bacteria changes either step by step (e.g., via consumption of nutrients) or unexpectedly due to some other influence. actions can occur such that all th bacteria in a area are killed or a group is isolated into a new part of the environment. They have the effect of perhaps destroying the chemotactic progress, but they also have the effect of support in chemotaxis, since spreading may place bacteria near good food sources. From a wide perspective, elimination and dispersal are parts of the population-level long-distance motile behavior. 


\subsection{System Design}

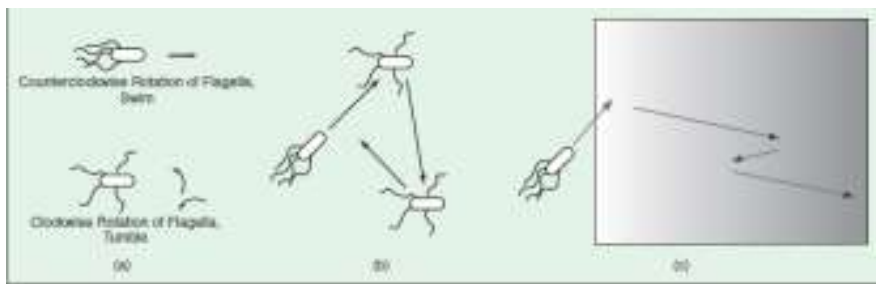

Fig 3.1 swimming, tumbling, chemo taxis behaviour of bacteria.

To explain how chemotaxis motions are generated, we must simply explain how the bacteriadecides how long to run,since from the above discussion we know what happens during a tumble or run. First, note with the intention of if an E. coli is in some substance that is neutral in the sense that it does not have food or noxious substances, and if it is in this medium for a long time (e.g., more than $1 \mathrm{~min}$ ), then the flagella will simultaneously alternate between moving clockwise and counterclockwise so that the bacterium will alternately tumble and run. This alternation between the two modes will move the bacterium, but in arbitrary directions, and this enables it to "search" for nutrients. For instance, in the isotropic homogeneous environment described above, the bacterium alternately tumbles and runs with the mean tumble and run lengths given above and at the speed that was given. If the bacteria are placed in a homogeneous concentration of serine (i.e., one with a nutrient but no gradients), then a variety of changes occurs in the characteristics of their motile behavior. For instance, mean run length and mean speed increase and mean tumble time decreases. They do still produce, however, a basic type of searching behavior; even though the bacterium has some food, it persistently searches for more. Suppose that we call this its baseline behavior. As an example of tumbles and runs in the isotropic homogeneous medium described above, in one trial motility experiment lasting $29.5 \mathrm{~s}$ there were 26 runs, the maximum run length was $3.6 \mathrm{~s}$, and the mean speed was about $21 \mathrm{Next}$, suppose that the bacterium happens to meet a nutrient gradient (e.g., serine). The change in the concentration of the food triggers a reaction such that the bacterium will spend large time swimming and less time tumbling..

I. Performance Result

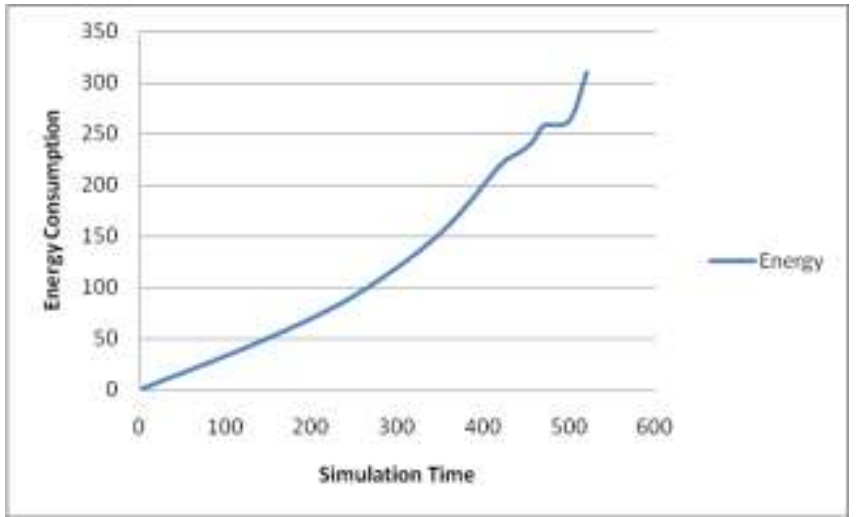

Fig:-4.1Simulation Time VS Energy Consumption

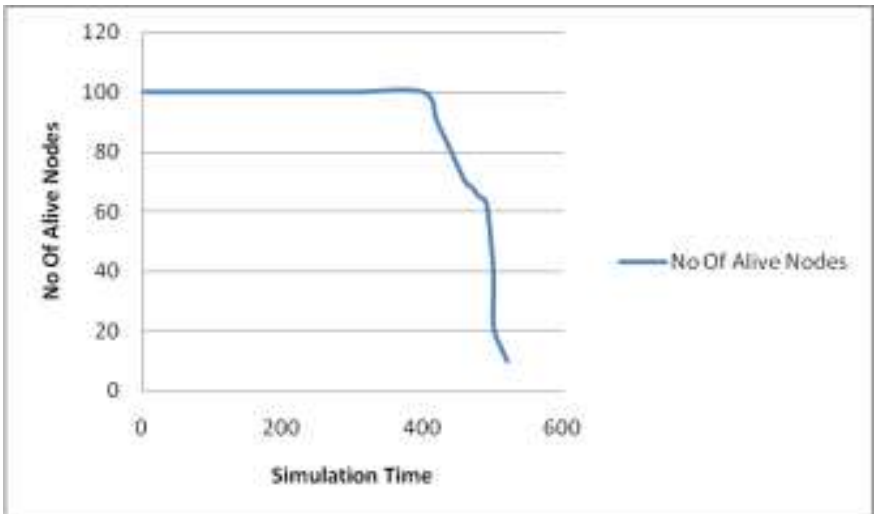

Fig:- 4.2 Simulation Time VS No.Of.Alive Nodes 


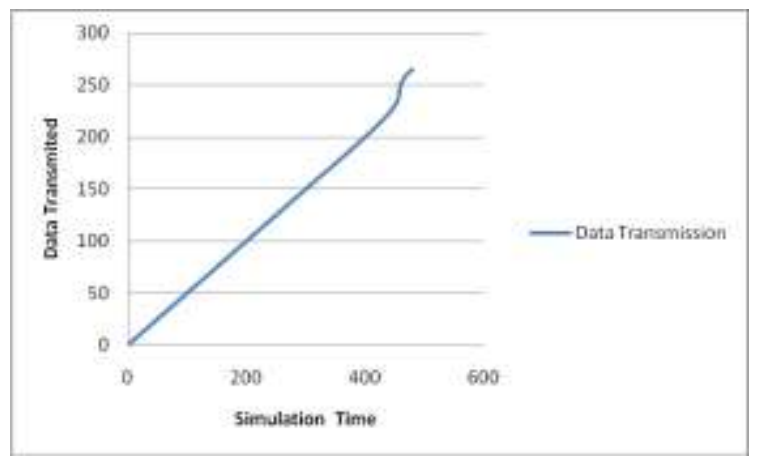

Fig:-4.3Simulation Time VS Data Transmission

\section{Conclusions}

The first contribution of the scheme is related to use of bacteria foraging algorithm firstly for wsns for enhancing network lifetime of sensor nodes. To validate the algorithm, simulations had been carried out using NS2. simulation results showed better performance of BFA as compared to other clustering protocols like leach,in terms of performance metrics like number of alive nodes and total energy dissipation in the system. BFA provides better lifetime for nodes compared to LEACH. It is also seen that BFA is able to provide $100 \%$ live nodes for maximum duration. leach provides a considerably higher lifetime compared to k-means clustering. In addition to dipping energy dissipation, leach successfully distributes energy-usage among every nodes in the network such that the nodes die arbitrarily and at essentially the same rate. BFA is self organizing and each node works independently. Provide efficient and scalable energy reduction.

\section{References}

[1] D. Stephens and J. Krebs, Foraging Theory. Princeton, NJ: Princeton Univ.Press, 1986.

[2] W. O’Brien, H. Browman, and B. Evans, "Search strategies of foraging animals,"Amer. Scientist, vol. 78, pp. 152-160, Mar./Apr. 1990.

[3] Giuseppe Anastasi, Marco Conti, Mario Di Francesco, Andrea Passarella, "Energy Conservation in Wireless Sensor networks: A Survey", Ad Hoc Networks 7 (2009) 537-568

[4] I. Banerjee, H. Rahaman, B. Sikdar, "UDDN: Unidirectional Data Dissemination via Negotiation", IEEE International Conference on Information Networking 2008,23-25 January, Pusan, Korea.

[5] Christopher Ho, Katia Obraczka, Gene Tsudik, and Kumar Viswanath, "Flooding for reliable multicast in multi-hop ad hoc networks", In Proceedings of the 3rd International Workshop on Discrete Algorithms and Methods for Mobile Computing and Communications (DIAL-M'99), 1999, pp. 64-71.

[6] Ming Liu, Jiannong Cao, Guihai Chen, and Xiaomin Wang, "An Energy-Aware Routing Protocol in Wireless Sensor Networks", Sensors 2009, vol. 9, pp. 445-462.

[7] Luis Javier García Villalba, Ana Lucila Sandoval Orozco, Alicia Triviño Cabrera, and Cláudia Jacy Barenco Abbas, "Routing Protocol in Wireless Sensor Networks", Sensors 2009, vol. 9, pp. 8399- 8421.

[8] E. Zanaj, M. Baldi, and F. Chiaraluce, "Efficiency of the Gossip Algorithm for Wireless Sensor Networks", In Proceedings of the 15th International Conference on Software, Telecommunications and Computer Networks (SoftCOM), Split-Dubrovnik, Croatia, September, 2007.

[9] Kelvin.M.Passino “ Biomimicry of bacterial foraging for distributed and optimization control”.IEEE control system magazine.2002.
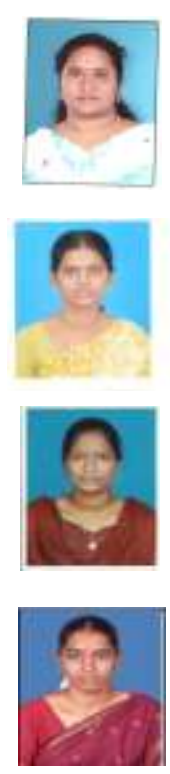

Mrs.A.RajeshwariGopinath, Assitant Professor in Department of Computer Science and Engineering,P.S.R Rengasamy College of Engineering for Women,Sivakasi.She has received her B.E degree in Computer Science and Engg in 2008 and M.Tech degree in Computer Science and Engineering in 2012 Kalasalingam University,Krishnan Kovil. She has published many papers in various fields. She is a member of ISTE.

Ms.V.VimalaDevi ,Assitant Professor in Department of Computer Science and Engineering,P.S.R Rengasamy College of Engineering for Women,Sivakasi.She has received her B.E degree in Computer Science and Engg in 2007 and M.E degree in Computer Science and Engineering in 2012 Anna University Chennai . She has published many papers in various fields. She is a member of ISTE.

Mrs.A.S.Lakshmi, Assitant Professor in Department of Computer Science and Engineering,P.S.R Rengasamy College of Engineering for Women,Sivakasi.She has received her B.E degree in Computer Science and Engg in 2004 and M.E degree in Computer Science and Engineering in 2012 Anna University Chennai . She has published many papers in various fields. She is a member of AIE.

Ms.N.Nagajothi ,Assitant Professor in Department of Computer Science and Engineering,P.S.R Rengasamy College of Engineering for Women,Sivakasi.She has received her B.E degree in Computer Science and Engg in 2009 and M.E degree in Computer Science and Engineering in 2012 Anna University Chennai . She has published many papers in various fields. She is a member of ISTE. 